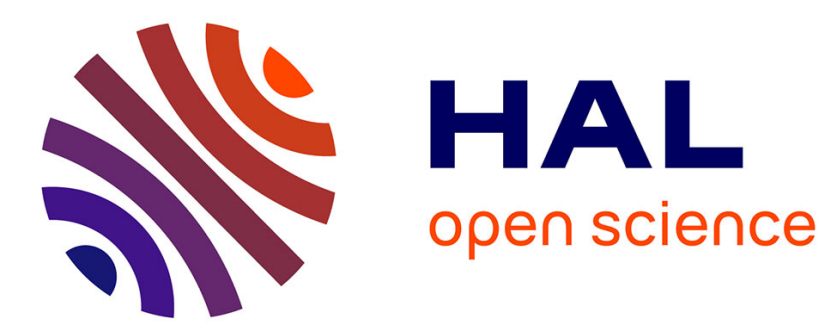

\title{
Frustration in polymers
}

\author{
M. Kléman
}

\section{To cite this version:}

M. Kléman. Frustration in polymers. Journal de Physique Lettres, 1985, 46 (16), pp.723-732. 10.1051/jphyslet:019850046016072300 . jpa-00232890

\section{HAL Id: jpa-00232890 https://hal.science/jpa-00232890}

Submitted on 1 Jan 1985

HAL is a multi-disciplinary open access archive for the deposit and dissemination of scientific research documents, whether they are published or not. The documents may come from teaching and research institutions in France or abroad, or from public or private research centers.
L'archive ouverte pluridisciplinaire HAL, est destinée au dépôt et à la diffusion de documents scientifiques de niveau recherche, publiés ou non, émanant des établissements d'enseignement et de recherche français ou étrangers, des laboratoires publics ou privés. 
Classification

Physics Abstracts

$61.30-61.40-61.70$

\title{
Frustration in polymers
}

\author{
M. Kléman \\ Laboratoire de Physique des Solides (*), Université Paris-Sud, \\ Bât. 510, 91405 Orsay Cedex, France
}

(Reçu le 25 avril 1985, accepté le 20 juin 1985)

\begin{abstract}
Résumé. - La configuration locale dans les polymères est le résultat d'une compétition entre effets entropiques et forces attractives. Ce type de frustration peut se représenter par un cristal d'espace courbé aux propriétés géométriques très proches de la représentation en espace courbé de phases bleues. Nous étudions dans ce cadre les ordres nématiques et cholestériques des cristaux liquides polymériques et suggérons des développements relatifs à l'ordre local des polymères fondus. Les paramètres pertinents de cette théorie sont le nombre de chaînes voisines d'une chaîne donnée et la longueur de persistance. On met en outre en évidence un intéressant isomorphisme entre les divers types d'empilement locaux denses de chaînes flexibles et les deltaèdres platoniciens.
\end{abstract}

\begin{abstract}
The local configuration of polymers results from a competition between entropy effects and attractive forces which provide a high density. The resulting frustration is studied geometrically in a curved space representation reminiscent of the curved space representation for blue phases. Within this frame work, we study the occurrence of nematic and cholesteric order and make some suggestions for further studies in molten polymers as a function of the number of neighbouring chains of a given chain and of the persistence length. An interesting isomorphism between locally densepacked strings of flexible chains and Platonic deltahedra is demonstrated.
\end{abstract}

\section{Introduction.}

Let us assign the term of frustration, popularized by G. Toulouse for spin-glasses, to the case in which the tendencies (Van der Waals forces, steric hindrance, entropy, etc...) which compete in the stability of some material do not succeed in building complete order at the molecular level because of geometrical impossibilities. Apart from spin-glasses, for which this term was first introduced, we know two other examples : the blue phases of chiral molecules, and the amorphous materials. In the first case the molecules are arranged in double twisted configurations which are stabilized locally, at a lower energy than the cholesteric states, by the saddle-splay energy term [1], but they cannot extend over all space because bend energy becomes prohibitive. The second case concerns a) metallic glasses : the tendency to build structures as dense as possible favours pentagonal order, an order which is clearly noncrystallographic in Euclidean space [2] ; b) covalent glasses : it is not

(*) Laboratoire associé au CNRS. 
possible to extend over space a local arrangement where all the angles between bonds are equal [3]. In all these cases frustration is given a geometrical representation by a " crystal " in a $3 \mathrm{~d}$ curved space, either spherical or hyperbolic; the interest of this representation is that, in contradistinction to the real case, all the competing interactions are satisfied everywhere and on all scales. The real system is obtained from the ideal curved one by introducing disclinations, either regularly disposed (in this case, translational order is recovered with a periodicity scaling with the distance between disclinations) or irregularly disposed. It is not yet clear which degree of universality such representations have, but their success in conceptualizing disorder at some scale is great enough to justify the search for a model of the same nature in other systems and, in particular, polymers.

This paper will deal with possible frustrated states in main-chain polymers; frustration is clearly documented in some cholesteric polymers of biological interest, as discussed below. We shall argue that its existence is worth considering in other liquid crystalline phases (biaxial nematics are an example), that it might be akin to self-assembly in biological systems made of mesogenic molecules, and that finally even molten and amorphous polymers might benefit by this new concept. After all, the Vogel-Fulcher law which rules transport processes in amorphous metals and which is considered as very characteristic of disorder was first discovered in polymers [4] ; similarly, the anomalous heat capacity observed at very low temperatures in amorphous metals and covalent glasses was also observed in polymeric systems [5].

(a)

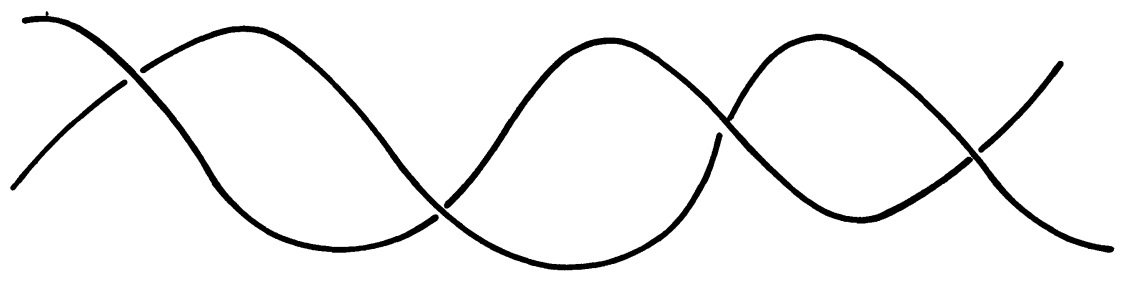

(b)

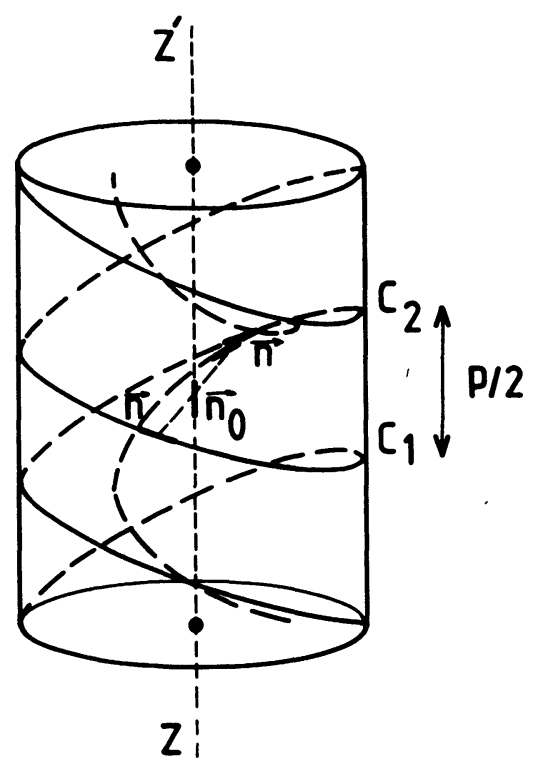

Fig. 1. - (a) Helical entanglement of two chains as the simplest example of a string made of strands. The chains are equidistant but not parallel. (b) The building block of the blue phase : molecules align along helices which entangle in the same way as in 1a. 
However, a difficulty remains with the definition of the forces which are at work in polymeric systems. In order to be able to define such forces unambiguously, let us consider molten polymers, which are supposedly in some thermodynamic state, rather than amorphous polymers, which are just frozen structures of molten polymers. We shall in fact restrict our considerations to two kinds of forces : the attractive forces, which tend to give the system a high density (under their action the macromolecules would crystallize or give a classical nematic or cholesteric phase) and the entropy forces, which tend to coil the macromolecule (it is these forces which dominate at high temperatures and are responsible for the disordered state). Of course, we limit our discussion to the case of long chains. Under the combined actions of these forces, we expect the coils of neighbouring chains to be locally equidistant. This suggests that the local state resembles that of $a$ string with many strands (Fig. 1a). There is therefore some local helicity, which has not to be mistaken for the helicity due to the chirality of the molecules, if any. The chirality of the molecules has a given sign, and it would give rise to a cholesteric phase with a definite pitch. On the other hand, the local helicity can be of either sign and its pitch variable. We shall see that in fact the local pitch is related to the local density and how this is so.

The model we propose is inspired by a theoretical study of the coexistence of $2 \mathrm{~d}$ and cholesteric orders we have made recently [6]. Such a problem arises with rigid polymers in solution, like DNA or PBLG. It is known that these phases display a local hexagonal order. DNA shows precholesteric phases [7], which might have some relationship with the blue phases of small mesogenic molecules (Fig. 1b), since the larger the ratio $\xi / p$ (coherence length over pitch), the larger the occurrence of a blue phase. Hence, in both cases, there are local configurations which manifest a compromise between incompatible trends. The description of this energetic competition in terms of order in a curved space is well known for blue phases : the relevant "crystal " is a double twisted director configuration in $S^{3}$ [8], the $3 \mathrm{~d}$ sphere in $4 \mathrm{~d}$ Euclidean space whose equation reads

$$
x_{0}^{2}+x_{1}^{2}+x_{2}^{2}+x_{3}^{2}=R^{2} \text {. }
$$

The field of $3 d$ unit vectors

$$
\mathbf{n}(\mathbf{x})=\frac{1}{R}\left(-x_{1}, x_{0}, x_{3},-x_{2}\right)
$$

satisfies such a double twist and is homogeneous throughout space $\mathrm{S}^{3}$, as well as in any field obtained by applying a constant rotation to all n's. The pitch of the twist is $+2 \pi R$. At different choice for the n's like

$$
\mathbf{n}(\mathbf{x})=\frac{1}{R}\left(-x_{1}, x_{0},-x_{3}, x_{2}\right)
$$

would change the sign of the pitch.

Let us call $\mathrm{V}^{*}(\mathrm{BP})$ the manifold $\mathrm{S}^{3}$ endowed with this homogeneous configuration of directions. BP stands here for "blue phase ", and the asterix indicates that the configuration is chiral. It can be shown that the lines of force of the director in $\mathrm{V}^{*}(\mathrm{BP})$ are equidistant great circles [9] of $S^{3} . V^{*}(B P)$ can now, therefore, be easily modified to represent a chiral phase with local order between long flexible molecules as follows. Select a finite number of lines of forces of the director in $\mathrm{V}^{*}(\mathrm{BP})$ at periodic equal distances; they represent those molecules and form a new configuration in $S^{3}$ which models a medium which is double twisted and $2 \mathrm{~d}$ ordered. Call $\mathrm{V}^{*}(2 \mathrm{~d})$ this new configuration in $S^{3}$; its pitch is still $p= \pm 2 \pi R$ (the sign of the pitch depends on the choice of a left-handed or right-handed set of equidistant circles).

It needs only a very minor modification in the interpretation of $\mathrm{V}^{*}(2 \mathrm{~d})$ to make it a good model for the frustration in a molten polymer. Let us call $V(M)$ two copies of $V^{*}(2 d)$, one left-handed, the other right-handed; each one can be used in turn to represent a local twist of the strands of the 
convenient sign. The purpose of the present paper is, first, to study the peculiar geometry of $2 \mathrm{~d}$ ordering of lines (great circles) in $\mathbf{S}^{\mathbf{3}}$, and second, to apply these results to systems of linear macromolecules, in particular molten polymers, nematics, and cholesterics.

\section{The geometrical properties of $\mathrm{V}(\mathrm{M})$.}

The results quoted above, viz. that there are families of equidistant great circles with doubletwist, are obtained easily when one uses for their demonstrations elliptic geometry, which is much simpler than spherical geometry, and not different locally [10].

Passing from spherical to elliptic geometry consists of identifying pairs of antipodal points on $\mathrm{S}^{\boldsymbol{n}}$. For example, the sphere $\mathrm{S}^{2}$ is replaced by the projective plane $\mathrm{P}^{2}$. Locally, lengths and angles are conserved, and any open set of points in $\mathrm{P}^{2}$ can be mapped isometrically on $\mathrm{S}^{2}$. But the geometry is simpler in the sense that straight lines always meet at one point, and at one point only, while they meet at two points on $\mathrm{S}^{2}$. Two points determine unique straight line on $\mathrm{P}^{2}$ (a pencil on $\mathrm{S}^{2}$ ). All straight lines are of the same finite length $\pi R$, while it is $2 \pi R$ in spherical geometry $\left(^{1}\right.$ ). The tilings of $\mathbf{P}^{2}$ are the same as those of $\mathrm{S}^{2}$, except that the number of polygons is divided by 2 .

Finally, there is duality between lines and points; to any point A corresponds a straight line $a$ whose points are all at a distance $\frac{\pi}{2} R$ from $\mathrm{A}$, such that any straight line passing through $\mathrm{A}$ is perpendicular to $a$. A is called the absolute pole of $a$.

All these geometrical properties, which are evident on $\mathrm{P}^{2}$, can be generalized very easily to $\mathrm{P}^{3}$ : all the perpendiculars to an (elliptic) geodesic plane a pass through a point $\mathrm{A}$, the absolute pole of $a$, and the locus of points at a distance $\frac{\pi}{2} R$ of a point $A$ is a plane $a$, the absolute polar plane of $A$. The two polar planes $a$ and $b$ of two points $A$ and $B$ cut along a line $L$ which is conjugate to $l$, the straight line determined by $a$ and $b$. Remember that $l$ and $\mathrm{L}$ are elliptic geodesic lines, and as such of finite length $\pi R$. Any point $\mathrm{c}$ on $l$ is at a distance $\frac{\pi}{2} R$ of any point $\mathrm{C}$ on $\mathrm{L}$, and any straight line $\mathrm{cC}$ which meets both $l$ and $\mathrm{L}$ cuts them at right angles. One can visualize $l$ and $\mathrm{L}$ as two entangled equal circles, one passing through the centre of the other, although this is a very incomplete visualization since the distance $\mathrm{cC}$ has to be measured in elliptic space, not along the Euclidean radii of these two entangled circles.

In elliptic geometry, the surface equidistant from a geodesic $l$ is a ruled surface $\Sigma_{l}$ of the second degree, with two sets of rectilinear generators, called a Clifford surface. There are very interesting properties of reciprocity here ; $\Sigma_{l}$ is also equidistant from $\mathrm{L}$, the line conjugate to $l$; any generator of $\Sigma_{l}$ is in turn the axis of a Clifford surface containing $l$ or L. A Clifford surface is a double surface of revolution about $l$ and $\mathrm{L}$; it can be represented by an anchoring (a torus), but is developable on the plane. The two sets of generators are equidistant from $l$ and $\mathrm{L}$, although not parallel to $l$ and $\mathrm{L}$. Any generator $\mathrm{g}$ is in skew position with respect to $l$ and $\mathrm{L}$ (Fig. 2). Let $d$ be the distance from $l$ to $\mathrm{g}$, measured along any common perpendicular $\Lambda ; \frac{\pi R}{2}-d$ is the distance from $\mathrm{g}$ to $\mathrm{L}$ along the same common perpendiculars, $R$ being the radius of curvature of $\mathrm{S}^{3}$ or $\mathrm{P}^{3}$. The angle between $\mathrm{g}$ and $l$ is $\theta=\frac{d}{R}$; similarly, the angle between $\mathrm{g}$ and $\mathrm{L}$ is $\frac{\pi}{2}-\theta=(\pi R / 2-d) / R$. Therefore, when one proceeds along $\Lambda$ from $l$ to $\mathrm{L}$, the Clifford parallels $\mathrm{g}$ (they may also be called paratactic lines) rotate about $\Lambda$ with a pitch $|p|=2 \pi R$. This rotation can be either clockwise $(p>0)$ or

$\left.{ }^{1}\right)$ These straight lines are great circles on $\mathrm{S}^{2}$ and « half " great circles on $\mathrm{P}^{2}$. The straight lines of $\mathrm{S}^{3}$ are great circles. 
counterclockwise $(p<0)$; there are therefore two sets of Clifford parallels, defined by the sign of the pitch. There are two Clifford parallels to $l$ and $\mathrm{L}$ at any point in space, each belonging to one of the sets, which are of course the sets of generators of the Clifford surface. Finally, each Clifford surface is defined by a distance $d$ to $l\left(\frac{\pi R}{2}-d\right.$ to $\left.\mathrm{L}\right)$ and an angle $\theta=\frac{d}{R}$. The angle between two generators is $2 \theta$.

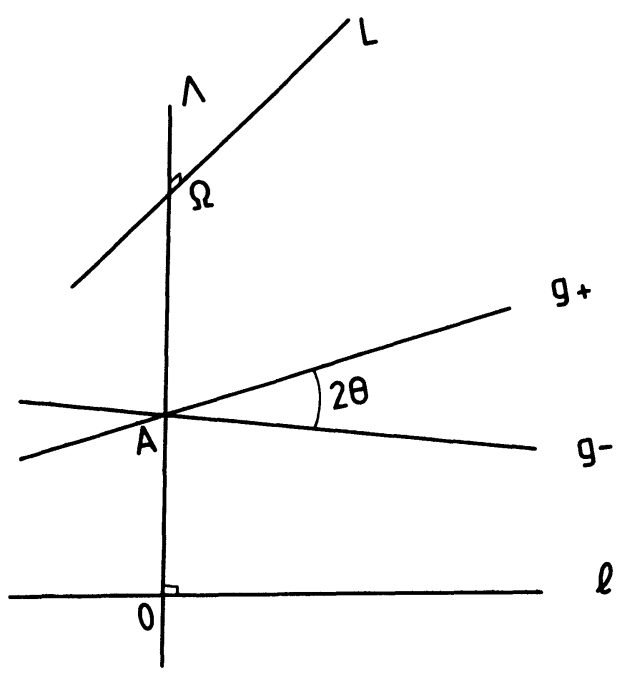

Fig. 2. - (a) $l$ and $\mathrm{L}$ are mutually polar lines in $\mathrm{P}^{3}$ (or $\left.\mathrm{S}^{3}\right) ; \mathrm{g}^{+}$and $\mathrm{g}^{-}$are equidistant to $l$ and $\mathrm{L}$ at a distance $\mathrm{OA}=d=R, \mathrm{~A} \Omega=\frac{\pi R}{2}-d \cdot \mathrm{g}^{-}$is a left Clifford parallel, $\mathrm{g}^{+}$is a right Clifford parallel ; all Clifford parallels to $l$ at a distance $d$ generate a ruled Clifford surface with two axes of revolution, $l$ and $\mathrm{L}$.

When going to $S^{3}$, the only difference is that lengths in space are duplicated. While the total length of a straight line is $\pi R$ in $\mathrm{P}^{3}$ (so that two points at distance $d$ are also at distance $\pi R-d$ ), it is $2 \pi R$ in $\mathrm{S}^{3}$. While the developed area of a Clifford surface is $\pi^{2} R^{2} \sin 2 \theta$ in $\mathrm{P}^{3}$, it is $4 \pi^{2} R^{2}$ $\sin 2 \theta$ in $\mathrm{S}^{3}$, each line developing a length $2 \pi R$. The relationship between a Clifford surface in $\mathrm{P}^{3}$ and $S^{3}$ is represented in figure 3.

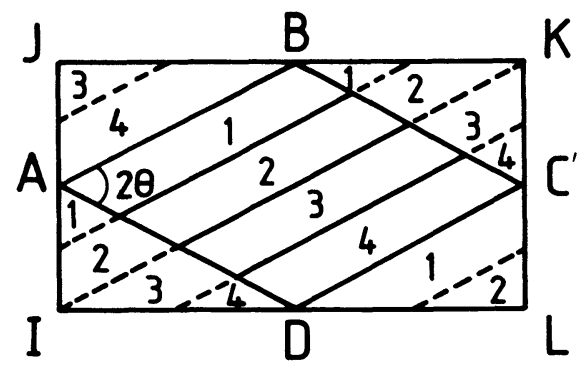

Fig. 3. - Clifford surface in $\mathrm{P}^{3}$ and its $\mathrm{S}^{3}$ extension; the rhombus $\mathrm{ABCD}$ is a planar development of a Clifford surface with two sets of generators (equidistant to $A B$ and to $A D$ ). $A B$ and $C D$ are identified in space, as well as $\mathrm{AD}$ and $\mathrm{BC}$. The $\mathrm{S}^{3}$ extension (by adding the antipodal surface) is represented by the rectangle IJKL, where IL (resp. IJ) and JK (resp. LK) are identified. Hence any generator of length $\pi R(\mathrm{AB}=\pi R)$ extends to a generator of length $2 \pi R$. We have also represented how a multiple banding parallel to one set of generators (here $n=4$ ) of the Clifford surface extends in $S^{3}$. 
Call $\mathrm{G}^{+}, \mathrm{G}^{-}$the two sets of Clifford parallels $\mathrm{g}^{+}, \mathrm{g}^{-}$to $l$ and $\mathrm{L}$. Set $\mathrm{G}^{+}$(say) is the curved space representation of the blue phase of Sethna et al. [8]. A unit vector, anywhere along $\mathrm{G}^{+}$, represents a director. Clearly, the integral curves of the directors are equidistant straight lines : the blue phase in curved space is a chiral phase, pitch $p$, made of paratactic (equidistant) molecules; it is nematic and cholesteric at the same time.

It is now easy to specialize to molecules with positional correlations between the straight lines of a set $\mathrm{G}$ in $\mathrm{S}^{3}$ or $\mathrm{P}^{3}$. For example, deciding that neighbouring lines (carrying molecules) of a given line $l$ are at a distance $d$ from $l$, they are necessarily on the corresponding Clifford surface, where they can be disposed of at will. An interesting case to investigate is when all the molecular alignments are equidistant, each line having $n$ neighbouring lines at a distance $d$. This is a problem which generalizes to $S^{3}$ the problem of maximum packing of cylinders in Euclidean space, where the solution is unique : the cylinders pack in a $2 d$ hexagonal network. In $S^{3}$, contrarily to our intuition, we shall find that there are many solutions, depending on the ratio $d / p$.

Consider a Clifford surface $\Sigma$ at a distance $d$ from $l$. Any line of set $\mathrm{G}^{-}$is cut by all the lines of set $\mathrm{G}^{+}$on $\Sigma$. Since the total length of a line is $\pi R$, and if $n$ is the number of neighbouring lines of $l$, the distance between equidistant lines of the set $\mathrm{G}^{+}$on $\Sigma$ is $u=\frac{\pi R}{n}$, this distance being measured along $\mathrm{g}^{-}$. Note that this result holds also in $\mathrm{S}^{3}$ (no doubling of $u$ ). Now the distance between neighbouring lines of the set $\mathrm{G}^{+}$is $d=R \theta$, and we can express $d$ as a function of $u$ in the right triangle (Fig. 4). We have (see Ref. [10]) :

$$
1=2 \sin \frac{u}{R} \cos \frac{d}{R}
$$

i.e.

$$
\cos 2 \pi \frac{d}{p}=\frac{1}{2 \sin \frac{\pi}{n}} .
$$

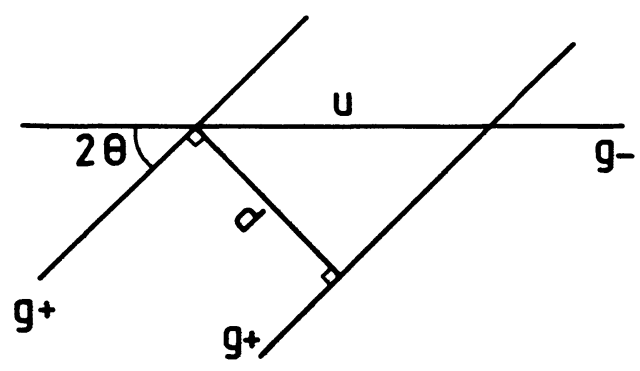

Fig. 4. - Trigonometric relations in a right angle triangle (see text).

The solutions with $n$ integer are summarized in table I, where $n_{0}=\frac{12}{6-n}$ is the total number of points on $\mathrm{S}^{2}$ representing flexible molecules.

Clearly, the vanishing of $d / p$ obtained for $n=6$ can be interpreted with $R$ becoming infinite. The case $n=6$ depicts therefore the classical case of the densest packing of cylinders of radius $d / 2$ in ordinary space. The cases $n=2,3,4$ and 5 depict the densest possible packings in spherical space $S^{3}$. The packing density is calculated as the ratio of the volume of the total number of molecules in $\mathrm{P}^{3}$ divided by the volume of $\mathrm{P}^{3}$. Standard calculations in elliptic geometry give :

$$
\begin{aligned}
& V\left(\mathrm{P}^{3}\right)=\pi^{2} R^{3} \\
& V(r)=\pi^{2} R^{3} \sin ^{2} \frac{r}{R}
\end{aligned}
$$


Table I. - Regular dense-packing in spherical space $\mathrm{S}^{3}$ of flexible chains in function of the number of neighbours $n$ of a given chain. $\theta_{\mathrm{rad}}$ : angle between two neighbouring chains; $p=2 \pi R$ : local pitch $; d$ : diameter of a chain $; n_{0}:$ total number of chains in $\mathrm{S}^{3}$.

\begin{tabular}{|c|c|c|c|c|}
\hline$n$ & $\theta_{\mathrm{rad}}=d / R$ & $d / p$ & $n_{0}$ & Packing density \\
\hline 2 & $\pi / 3$ & $0.1666 \ldots$ & 3 & 0.75 \\
3 & $0.9553 .$. & $0.1520 \ldots$ & 4 & $0.8453 .$. \\
4 & $\pi / 4$ & 0.125 & 6 & $0.8787 .$. \\
5 & $0.5536 .$. & $0.0881 .$. & 12 & $0.8961 .$. \\
6 & 0 & 0 & $\infty$ & $0.9069 .$. \\
\hline
\end{tabular}

where $V(r)$ is the volume of the region « inside » the Clifford surface located at a distance $r$ of one of its axes of revolution (the volume « outside " is $\left.V\left(\mathrm{P}^{3}\right)-V(r)=V\left(\frac{\pi R}{2}-r\right)\right)$.

The calculation of the number $n_{0}$ of molecules in contact in $\mathrm{S}^{3}$ reveals an astonishing relationship between the structure of the densest packings in question and the periodic tilings of the sphere $S^{2}$. We use the fact that each Clifford surface at a distance $r=R \theta$ of a line $l$ can be represented on $\mathrm{S}^{2}$ by a small circle of colatitude $2 \theta$, the pole representing the line $l$. The polar line of $l$, $L$, is represented by the other pole of $S^{2}$ (Fig. 5). Each point of $S^{2}$ on a small circle represents a generator of the Clifford surface, either left or right according to the choice. Hence all paratactic lines $\mathrm{g}^{+}$, say, of $\mathrm{P}^{3}$ (and their extension to great circles in $\mathrm{S}^{3}$ ) are in one to one correspondence with the points of $S^{2}$, and their distance in $S^{3}$ is directly measured by half the angle which separates them on $S^{2}$.

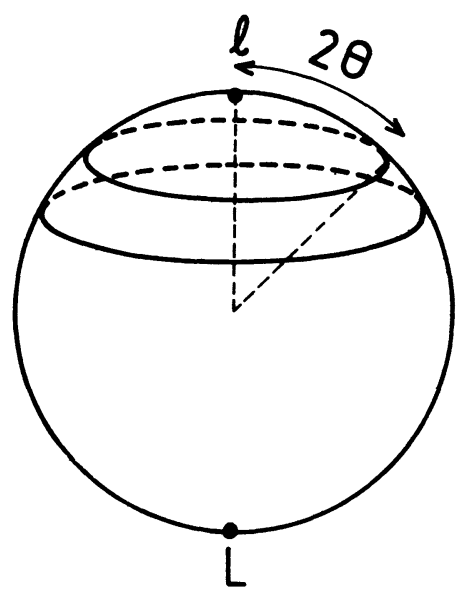

Fig. 5. - Representation on $S^{2}$ of a set of paratactic lines in $S^{3}$. Each small circle represents a Clifford surface.

These results are nothing else than a rephrasing of the famous Hopf fibering theorem [11] of the sphere $S^{3}$ by great circles, with basis $S^{2}$. Its use is now obvious : representative points on $S^{2}$ of the molecules in closest packing are on the vertices of a regular deltahedron of $S^{2}$. For $n=5$, it is the icosahedron, i.e. $n_{0}=12$; for $n=4$, it is the octahedron, i.e. $n_{0}=6$; for $n=3$ it is the tetrahedron $\left(n_{0}=4\right)$; for $n=2$, it is a set of 3 points at mutual distance $2 \theta=\frac{2 \pi}{3}$, on a great circle $\left(n_{0}=3\right)$. 
Note that neither the cube, nor the dodecahedron appear in our list : it is easy to see that they would not correspond to our model of closest packing in which there is a central line, represented by one (or both) of the poles. Clearly the cube and the dodecahedron correspond to other possibilities, without central line, and can easily be calculated too, directly on the $S^{2}$ representation (Table II).

Table II. - Regular (but not dense) packing of flexible chains in spherical space $\mathrm{S}^{3}$

\begin{tabular}{|l|c|c|c|c|c|}
\hline & $\cos \theta$ & $\theta$ rad. & $d / p$ & $n_{0}$ & $\begin{array}{c}\text { Packing } \\
\text { density }\end{array}$ \\
\cline { 2 - 6 } Cube & $\sqrt{2 / 3}$ & $0.6155 .$. & $0.09796 .$. & 8 & $0.7340 .$. \\
\cline { 2 - 6 } Dodecahedron & $\tau / \sqrt{3}$ & $0.3649 .$. & $0.05807 .$. & 20 & $0.6583 .$. \\
\hline
\end{tabular}

$\tau=(1+\sqrt{5}) / 2$

Finally, any anisotropic distribution of paratactic molecules can be represented on the sphere $\mathrm{S}^{2}$, and similarly its structural properties easily calculated.

The problem of « best " packing of $n_{0}$ points on a sphere (defined as follows : the least distance between points should be as great as possible) is known as Tammes problem [12]. Apart from the cases of table I, solutions are known for $n_{0}=7,8,9,10,11$ and 24. Their images on $S^{2}$ are not regular ; for example, for $n_{0}=8$ the best arrangement is the antiprism $(3,3,3,4)$, with 3 triangles and one square at each vertex (for this notation, see Ref. [12], p. 108).

\section{Back to space of physical phenomena.}

The geometry of strings and strands is isomorphic to the geometry of lines in $\mathrm{S}^{\mathbf{3}}$, in a mapping of a Clifford volume to real space which preserves the local configuration but introduces distortions : the Clifford surface $\theta=\pi / 4$ (the spherical torus, in $S^{3}$ ) divides $S^{3}$ into two equal volumes; if the spherical torus is developed in real space, the inside volume is changed from $2 \pi^{2} R^{3}$ in $S^{3}$ to $\frac{\pi^{2} R^{3}}{\sqrt{2}}$ in real space. This is an enormous distortion, and the mapping cannot be done indeed without also changing the lengths on the Clifford surfaces. We shall not enter into the details of this mapping $\left({ }^{2}\right)$ and just satisfy ourselves with its possibility. Note, in support of the fact that the spherical torus has already been used in the context of dense random packing of spheres [13], that the arrangement of tetrahedra of hard spheres inside the spherical torus shows helicity, and that a helicity has been indeed recognized in hand-made models of hard spheres [14] and correlated to the geometry of the spherical torus $[13,15]$.

In general, a string with its strands is therefore an inulge in flat space of a part of $V^{*}(2 d)$; locally we have string-like domains, each of them contaning less than, but of the order of, $n_{0}$ molecules. $n_{0}$ can be either of the order of the number of neighbours $n$, like in the Platonic models, or much larger, and in the latter case the $S^{2}$ representation contains a (necessarily) non-homogeneous distribution of points, whose number $n_{0}$ scales like the area of $S^{2}$, for instance. In both

$\left({ }^{2}\right)$ This will be discussed in a forthcoming paper. 
cases the string-like domains are separated by disclinations (as in [2]) or/and by walls (for chiral molecules). We shall argue that the case $n_{0}$ large occurs for long persistence lengths of chiral molecules.

The relevant physical parameters to be used in applying the former geometrical considerations are :

- the number of neighbours or, equivalently, the local pitch $p$ or the density in spherical space, related of course to the density in real space.

- the total number of molecules, of the order of $n_{0}$, involved in an elementary domain, of transverse correlation length $\xi$ which must be of the order of $p$.

- the persistence length $l_{\mathrm{p}}$.

- the cholesteric pitch $p^{*}$ (for chiral molecules).

Clearly, if the persistence length $l_{\mathrm{p}}$ is larger than $p$, the model does not apply. This would be the case of a true cholesteric with simple twist ( $p^{*}$ finite) or a true nematic with $n \sim 6$ ( $p^{*}$ infinite). It would also be the case of a polymer melt with ideal chains in the sense of Flory, since by definition there is no correlation at all between neighbouring chains, i.e. $p=0$. The closest approximation to ideal chains, in our model, is the case $n=2, n_{0}=3$ (Table I), but it is also the case where the spherical image is at farthest from reality, since the mapping to real space would bring so large distortions.

3.1 NON-CHIRAL MOLECULES. - If $l_{\mathrm{p}}<p$, our model might help in conceiving various kinds of molten or amorphous polymers whose chains show up some difference with ideality. This difference is manifest only at a local scale, of the size $p<L$, where $L$ is the extended chain length. Our model of local coiling is then superimposed on Flory's ideal chain; it leads to some decrease of entropy, but also to a decrease of internal energy since locally the chains are equidistant. Finally, it provides a model for the origin of free volume, since the local density in a coil is always smaller than in a packing of parallel chains.

Of course, one might expect interesting applications of the model for nematics with $5<n<6$, or with anisotropic neighbourhood (biaxial nematics). Such systems might have very long transverse correlation lengths $\xi \sim p$, with $n_{0}$ large. The defects separating domains can be disclinations or walls.

Relevant experiments would be in looking for such correlations above and below $T_{\mathrm{c}}$ in biaxial and non-biaxial nematics.

3. 2 Chiral molecules. - The $S^{2}$ model endowed with a large number of points $n_{0}$, best distributed in the sense of Tammes, but not regularly if $n_{0}$ has no Platonic value (Table I), represents a large pitch $p^{*}$ double twisted structure with $n$ smaller than 6 . If $n=6$ precisely, the system is of infinite pitch ( $p^{*}=\infty:$ nematic). If the proper pitch $p^{*}$ has a finite value, then $n \lesssim 6$, and we have to differentiate various possibilities as a function of $l_{\mathrm{p}}$, the persistence length. If $p^{*}$ is macroscopically large, this means that the Platonic double twist model does not work, i.e. $l_{\mathrm{p}}$ is large compared to a local (Platonic) pitch $p$. The relevant parameters therefore become the proper pitch itself $p^{*}$ (for a given $l_{\mathrm{p}}$ ) and the mean number of neighbours $n$ (i.e. the local pitch $p$ ). If the number of neighbours is of the order of 5 , it is possible to build a $S^{2}$ model with $n_{0}$ large (the mean number of neighbours $n$ for $n_{0}>20$, say, is always between 5 and 6, since the neighbouring space of a given chain becomes flatter when $n_{0}$ increases). Therefore one can obtain a macroscopic pitch $p^{*}$ with double twist like in blue phases or chromosomes.

If the number of neighbours is smaller than $5, n_{0}$ cannot reach a larger value than 20 , say. Therefore either the system builds a multistrand with $n_{0}$ elementary strands, and at larger scale one could obtain a structure with double twist, or one obtains a simple cholesteric texture, the $S^{2}$ model becoming irrelevant. Note that since $n$ is small, the neighbourhood of a polymeric molecule in a cholesteric is anisotropic (biaxial). 
For $n<5$, the choice between a multistrand structure or a simple cholesteric depends crucially on the transverse correlation length, which is of the order of the persistence length. If $l_{\mathrm{p}}$ is smaller than the multistrand lateral size $p\left(n_{0}\right)$, one can expect the double twist to be stabilized; if not, once again, the simple cholesteric phase would take place.

Summing up our results (at constant large $l_{\mathrm{p}}$ ) in terms of varying pitch $p^{*}$, one can expect nematics for $p^{*}$ infinite $(n=6)$, double twist for $p^{*}$ intermediate $(n=5)$ and cholesterics for $p^{*}$ smaller $(n=5,4)$.

But if now we consider the effect of a varying $l_{\mathrm{p}}$, we expect that for large $l_{\mathrm{p}}$ we have the nematic phase $(n=6)$, for intermediate $l_{\mathrm{p}}$ the double twist $(n=5)$ and for small $l_{\mathrm{p}}$ the cholesteric one. These results go in the same sense as the Landau theory [16] results if we interpret the scaling parameter $\kappa$ of this theory as the ratio $l_{\mathrm{p}} / p^{*}$.

\section{Acknowledgments.}

We have benefitted from fruitful discussions with Prof. J. Friedel.

\section{References}

[1] Meiboom, S., Anderson, P. W. and Brinkman, W. F., Phys. Rev. Lett. 46 (1981) 1216, and erratum in Phys. Rev. Lett. 25 (1981) 1656.

[2] KlÉman, M. and Sadoc, J. F., J. Physique Lett. 40 (1979) L569.

[3] Kléman, M. and Donnadieu, P., Defects in hyperbolic tessellations, Philos. Mag., in the press.

[4] Kovacs, A. J., Adv. Polym. Sci. 3 (1963) 394.

[5] Zeller, R. C. and PoHL, R. O., Phys. Rev. B 4(1971) 2029.

Stephens, R. B., Phys. Rev. B 8 (1973) 2896.

[6] KlÉman, M., Coexistence of cholesteric and 2d order, J. Physique, in press.

[7] Livolant, F., " La structure cristalline liquide de l'ADN in vivo et in vitro ». Thèse d'Etat, Paris (1984).

[8] Sethna, J. P., Wright, D. C. and Mermin, N. D., Phys. Rev. Lett. 51 (1983) 467.

[9] The equation of a great circle in $S^{3}$ is obtained as the common intersection of $S^{3}$ with two hyperplanes passing through the origin.

[10] CoXETER, H. S. M., Non Euclidean Geometry (Univ. of Toronto Press) 1968.

SOMMER Ville, D. Y. M., Non Euclidean Geometry (Dover) 1958.

[11] SteEnrod, N., The topology of fibre bundles (Princeton Univ. Press) 1974.

[12] Fejes Toth, L., Regular figures (Pergamon Press) 1964.

[13] SADOC, J. F. and MosserI, R., in Amorphous materials : modelling of structure and properties, V. Vitek ed., Metal Soc. of AIME (1983) p. 111.

[14] FinNeY, J. L., Nature 266 (1977) 309.

[15] Nelson, D. R. and Widom, M., Nucl. Phys. B 240 (1984) 113.

[16] HoRnreich, R. M., Bath-Sheva Conference, Jerusalem (oct. 1984).

Hornreich, R. M. and Shtrikman, S., J. Physique 41 (1980) 335. 